\title{
Demonstration of 80 Gbps NRZ-OOK Electro-Absorption Modulation of InP-on-Si DFB Laser Diodes
}

\author{
Mahmoud Shahin ${ }^{1, *}$, Javad Rahimi Vaskasi ${ }^{1}$, Joris Van Kerrebrouck ${ }^{2}$, Amin Abbasi ${ }^{1}$, Kasper Van Gasse ${ }^{1}$, \\ Muhammad Muneeb ${ }^{1}$, Laurens Breyne ${ }^{1,2}$, Peter Ossieur ${ }^{2}$, Xin Yin ${ }^{2}$, Johan Bauwelinck ${ }^{2}$, Gunther \\ Roelkens ${ }^{1}$, and Geert Morthier ${ }^{1}$ \\ ${ }^{1}$ Photonics Research Group, Department of Information Technology, Ghent University - imec, Ghent, Belgium \\ ${ }^{2}$ IDLab, Ghent University, imec, Gent, Belgium \\ *Mahmoud.Shahin@ugent.be
}

\begin{abstract}
High-speed electro-absorption modulation of a heterogeneously integrated InP-on-Si DFB laser diode is used for the transmission of an $80 \mathrm{Gbps}$ NRZ-OOK signal over $2 \mathrm{~km}$ of NZDSF link below the hard-decision forward-error-correction threshold. (C) 2019 The Author(s) OCIS codes: (140.3490) Lasers, distributed-feedback, (130.0250) Optoelectronics, (060.4510) Optical communications.
\end{abstract}

\section{Introduction}

Heterogeneous integration of III-V membranes on silicon, or silicon-on-insulator, is regarded as one of the few viable approaches to realize integrated sources for silicon photonics. A good candidate for optical datacommunication applications is the distributed-feedback (DFB) laser diode, thanks to its stable single mode behavior with large side mode suppression. A characteristic of great importance for data-communication is the maximum modulation speed of the transmitters. In recent years, several state-of-the-art results have been reported for both monolithic InP devices and heterogeneously integrated InP-on-Si devices. These were realized using different modulation schemes: non-return-to-zero on-off-keying (NRZ-OOK), 4-level pulse amplitude modulation (PAM-4) and electrical duobinary (EDB). As an example, a $100 \mathrm{Gbps} \mathrm{NRZ} \mathrm{GeSi} \mathrm{electro-absorption} \mathrm{modulator} \mathrm{implemented}$ on a silicon photonic platform was shown in [1]. For III-V-on-silicon devices, 56 Gbps NRZ-OOK electroabsorption modulation was demonstrated from both sides of the laser [2]. In [3], an InP distributed Bragg reflector (DBR) laser was used for the generation of 112 Gbps PAM-4. Furthermore, an InP-based double-side electroabsorption modulated DFB laser has been used for 2 x 56 Gbps PAM-4 [4]. For III-V-on-silicon devices, 25 Gbaud PAM-4 was demonstrated [2], and the same design was used for 100 Gbps EDB [5]. Although the bandwidth of direct modulation can be relatively high [3], modulation at high frequencies often comes with a large chirp. Electroabsorption modulation suffers less from this problem and its bandwidth is limited only by the RC-constant of the modulator. In this paper, we report on the NRZ-OOK electro-absorption modulation of a heterogeneously integrated III-V-on-silicon DFB laser at $80 \mathrm{Gbps}$. We briefly discuss the design and fabrication of the externally modulated laser diode (EML). We also show that the bit-error-rate (BER) is substantially below the $7 \%$ hard-decision forwarderror-correction (HD-FEC) threshold, both in back-to-back configuration as well as after transmission over $2 \mathrm{~km}$ of non-zero dispersion-shifted-fiber (NZ-DSF).

\section{Design and fabrication}

The top-view of the EML structure and the InP epitaxial layer stack design parameters are shown in Fig. 1. The taper structure used for coupling the light from the III-V-on-silicon DFB laser to the silicon waveguide is used as an EAM. The device is similar to that of [2], but with each taper section cut in two halves. The sections are electrically isolated from each other during fabrication, by wet-etching the top p-InGaAs contact layer, and dry-etching around $200 \mathrm{~nm}$ of the p-InP layer. The length of the etched region was $30 \mu \mathrm{m}$ instead of $7 \mu \mathrm{m}$ in [2]. High resistance between the sections minimizes the leakage current from one section to the other. In our laser, the resistance between the EAM and the laser section is $6 \mathrm{k} \Omega$.

The active laser section is $500 \mu \mathrm{m}$ long and $2 \mu \mathrm{m}$ wide. The EAM sections are each $50 \mu \mathrm{m}$ long. The full taper length is around $150 \mu \mathrm{m}$ long. The width of the taper goes from $2 \mu \mathrm{m}$ down to $0.7 \mu \mathrm{m}$. The InP epitaxial structure is
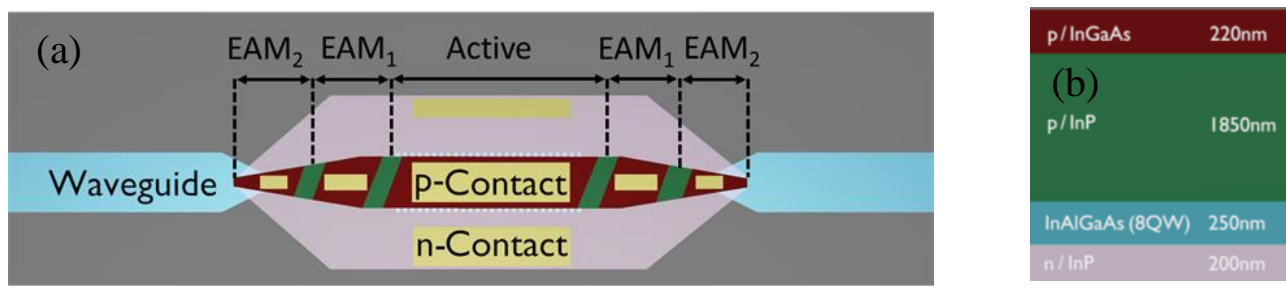

Fig. 1. (a) Top-view of the fabricated InP-on-Si EML showing the III-V-on-Si DFB and tapers used as EAM, and (b) the InP epitaxial layer stack design parameters. 
bonded on a $400 \mathrm{~nm}$ thick Si waveguide ( $3.5 \mu \mathrm{m}$ wide) that has a $500 \mu \mathrm{m}$ long DFB grating etched $190 \mathrm{~nm}$ deep, with a period of $248 \mathrm{~nm}$ and duty cycle of $50 \%$. A quarter-wave shifted section is located in the center of the grating. The detailed fabrication steps are reported in [6].

In principle, either of the EAM sections can be used to modulate the light. However, in the first EAM section, the light is confined more to the III-V waveguide, while in the second EAM section, the light is confined more to the silicon waveguide. Therefore, more light will be absorbed when reverse biasing the first EAM section, and a higher extinction ratio is expected. In this paper, we focus on modulating the first EAM section, while not pumping the second EAM section. If the lasing wavelength is close enough to the bandgap wavelength, reverse biasing of this section will result in a significant extinction with low insertion loss.

\section{Characterization}

The measured 3-dB modulation bandwidth of the device was found to be $33 \mathrm{GHz}$. Data transmission experiments were performed to investigate the large signal modulation performance of the EML. An electrical signal is generated with a Keysight M8196A Arbitrary Waveform Generator (AWG). This electrical signal is amplified by a $50 \mathrm{GHz}$ RF electrical amplifier (SHF-S807). The data signal is superimposed on a negative bias voltage using a bias tee. An L-band Erbium Doped Fiber Amplifier (EDFA) is used to boost the optical signal. The amplified spontaneous emission from that EDFA is filtered out using a variable-bandwidth optical tunable filter. The resulting optical signal is fed to the photodetector, and finally, the output electrical signal is fed to a Keysight DSAZ634A Real-Time Oscilloscope. A raised-cosine filter with $\alpha=0.1$ is used to shape the transmitted pulse. Using the built-in channel deembedding function of the AWG, transmission of a 80 Gbps NRZ-OOK Pseudorandom-Binary-Sequence (PRBS) with a word length of $2^{7}-1$ was verified, as shown in Fig. $2(a, b)$ for back-to-back configuration, and after transmission over a $2 \mathrm{~km}$ long NZ-DSF link, respectively.

The voltage swing applied to the EAM is about $1.8 \mathrm{Vpp}$. The stage temperature was fixed at $15^{\circ} \mathrm{C}$. The laser was operating at a bias current of $49 \mathrm{~mA}$ and an EAM reverse bias of $-0.7 \mathrm{~V}$. The lasing wavelength was $1570.6 \mathrm{~nm}$. The optical bandwidth of the optical tunable filter was $1 \mathrm{~nm}$. The received optical power was $5 \mathrm{dBm}$ (note that no transimpedance amplifier was present at the receiver). The recoded BER was around $1.5 \cdot 10^{-4}$, which is below the KP4FEC threshold. Measurement of the BER vs. received power for this laser is shown in Fig. 2 (c). 80 Gbps PRBS transmission with a word length of $\left(2^{7}-1\right)$ with a BER lower than the $7 \%$ HD-FEC threshold is possible for a received power less than $0 \mathrm{dBm}$.
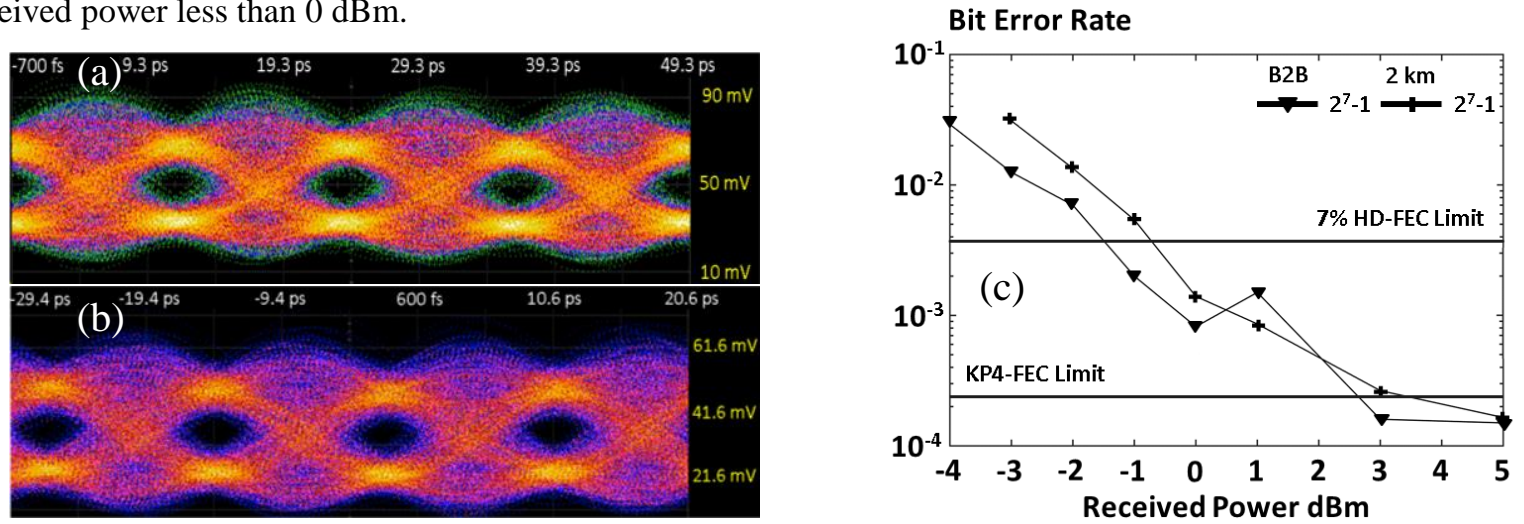

Fig. 2. Sub-HD-FEC transmission at $80 \mathrm{Gbps}$ (a) in back-to-back configuration, and (b) with $2 \mathrm{~km} \mathrm{NZ-DSF} \mathrm{link,} \mathrm{for}$ a PRBS pattern of length $2^{7}-1$. (c) Bit-error-rate vs. received power showing sub-HD-FEC operation for $80 \mathrm{Gbps}$ for

\section{Conclusions}

a received power below $0 \mathrm{dBm}$.

Electro-absorption modulation of InP-on-Si DFB laser diodes is demonstrated. Transmission of an 80 Gbps NRZOOK signal is shown for both back-to-back configuration and for a $2 \mathrm{~km}$ long NZ-DSF link between the transmitter and the receiver, with a BER below the 7\% HD-FEC threshold.

\section{References}

[1] J. Verbist et al., "Real-time $100 \mathrm{~Gb} / \mathrm{s} \mathrm{NRZ} \mathrm{and} \mathrm{EDB} \mathrm{transmission} \mathrm{with} \mathrm{a} \mathrm{GeSi} \mathrm{electro-absorption} \mathrm{modulator} \mathrm{...,"} \mathrm{JLT,} \mathrm{36(1),} \mathrm{(2018).}$

[2] A. Abbasi et al., "III-V-on-Silicon C-band High-Speed Electro-Absorption Modulated DFB Laser," JLT, 36(2), (2018).

[3] Y. Matsui et al., "55 GHz Bandwidth Distributed Reflector Laser," Journal of lightwave technology, 35(3), (2017).

[4] M. Theurer et al., " $2 \times 56$ GB/s From a Double Side Electroabsorption Modulated DFB Laser and Application ...," JLT, 35(4), (2017).

[5] A. Abbasi et al., "100-Gb/s Electro-Absorptive Duobinary Modulation of an InP-on-Si DFB Laser," IPTL, 30(12), pp. 1095-1098, (2018).

[6] G. Roelkens et al., "III-V-on-Silicon Photonic Devices for Optical Communication and Sensing," Photonics (3), (2015). 